\title{
Aplicação de redes neurais recorrentes na previsão de geração eólica
}

\section{Application of recurrent neural networks in forecasting wind generation}

Jandrei Sartori Spancerski ${ }^{1}$, José Airton Azevedo dos Santos ${ }^{2}$

\section{RESUMO}

O presente trabalho tem como objetivo avaliar modelos, baseados em redes neurais recorrentes, para a previsão da geração da usina eólica de Praia Formosa. A base de dados, disponibilizada pelo Operador Nacional do Sistema Elétrico (ONS), apresenta uma série histórica da geração eólica, da usina de Praia Formosa no Ceará, no período entre 2011 e 2020. Modelos de previsão, baseados em Redes Neurais LSTM (Long Short-Term Memory) e GRU (Gated Recurrent Unit), foram implementados na linguagem Python. Resultados obtidos, dos dois modelos, foram comparados. Verificou-se, para um horizonte de seis meses, que o modelo GRU apresentou um melhor desempenho que o modelo LSTM.

Palavras-chave: LSTM. GRU. Geração eólica.

\section{ABSTRACT}

The present work aims to evaluate models, based on recurrent neural networks, for forecasting the generation of the Praia Formosa wind power plant. The database, made available by the National Electric System Operator (ONS), presents a historical series of wind generation, from the Praia Formosa plant in Ceará, in the period between 2011 and 2020. Forecasting models, based on LSTM Neural Networks (Long Short-Term Memory) and GRU (Gated Recurrent Unit), were implemented in the Python language. Results obtained from the two models were compared. We found, for a six-month horizon, that the GRU model performed better than the LSTM model

Keywords: LSTM. GRU. Wind generation.
${ }^{1}$ Discente do Programa de PósGraduação em Tecnologias Computacionais para Agronegócio

Universidade para 0 Federal do Paraná (UTFPR). Email: jandreisst@gmail.com

${ }^{2}$ Doutor em Engenharia Elétrica. Docente do Programa de PósGraduação em Tecnologias Computacionais para

Agronegócio

Universidade

(PPGTCA). elará (UTFPR). Email: airton@utfpr.edu.br 


\section{INTRODUÇÃO}

A energia elétrica tem um papel importante na economia mundial. É considerada como um dos indicadores do nível de qualidade de vida e do desenvolvimento econômico. As pessoas dependem, direta ou indiretamente, da eletricidade e as indústrias a utilizam como um fator de produção (SANTOS; CHAUKOSKI, 2020).

Contudo, com o consumo crescendo continuamente, o fornecimento de energia elétrica é um dos grandes problemas da atualidade. A produção de energia, por meio de combustíveis fósseis, produz um efeito negativo sobre o meio ambiente. Uma alternativa, ao uso destes combustíveis, é a energia eólica. A energia eólica é uma fonte de energia limpa que não emite gases de efeito estufa. As usinas eólicas possuem um tempo curto de instalação, quando comparadas com as usinas hidráulicas (ARAÚJO; GOMES, 2012).

A geração eólica, devido à diminuição dos seus custos, tem-se mostrado competitiva tanto na instalação quanto nos equipamentos. Sendo uma das grandes apostas para os problemas energéticos mundiais (ARAÚJO; GOMES, 2012).

A produção de energia elétrica, no Sistema Interligado Nacional, é obtida por um sistema hidro-termo-eólico de grande porte, com predominância de usinas hidrelétricas (ONS, 2020, COELHO, 2018).

Segundo Lins e Torres (2020), a fonte hidráulica, na projeção da matriz brasileira (2019/2024), será a mais significativa, com 62,1\% de participação na potência instalada, seguida da fonte eólica com 11,1\%. Portanto, a participação da geração eólica na matriz brasileira é muito representativa, tendo papel fundamental no nordeste.

A previsão de geração eólica de uma usina, devido a dependência da velocidade do vento incidente nos aerogeradores, é de difícil determinação, embora seja imprescindível para o planejamento do setor elétrico. Métodos de previsão, de séries temporais, podem ser utilizados para avaliar a geração de energia elétrica ao longo do tempo.

Neste contexto, este trabalho tem como objetivo avaliar modelos, baseados em redes neurais recorrentes, para a previsão da geração da usina eólica de Praia Formosa no Ceará.

O artigo é organizado da seguinte maneira. Na Seção 2 é apresentada a metodologia utilizada na previsão da geração da usina eólica. Na Seção 3 são apresentados os resultados obtidos da aplicação desta metodologia. Comentários finais e conclusões, na Seção 4, finalizam o artigo. 


\section{MATERIAIS E METODOS}

Nesta seção aborda-se a metodologia utilizada para realizar essa pesquisa, detalhando os métodos propostos para realização dos experimentos, bem como as técnicas utilizadas.

\section{Redes Recorrentes:}

Redes Neurais Artificiais Recorrentes têm sido utilizadas, com sucesso, em tarefas de predição e modelagem de séries temporais. Redes recorrentes LSTMs contêm unidades especiais chamadas de células de memória (Figura 1). Cada célula de memória, denominada de neurônio da rede LSTM, contém três portas, uma porta de entrada (input gate) que determina se deve deixar ou não entrar uma nova entrada, uma porta de esquecimento (forget gate) que exclui informações que não são importantes e uma porta de saída (output gate) que decide quais informações devem ser enviadas. Estas portas gerenciam o fluxo de dados da rede neural (YADAV et al., 2020, HAYKIN, 2001; BASTIANI et al., 2018; GRAVES, 2014, NELSON et al., 2017; ZAO, 2017).

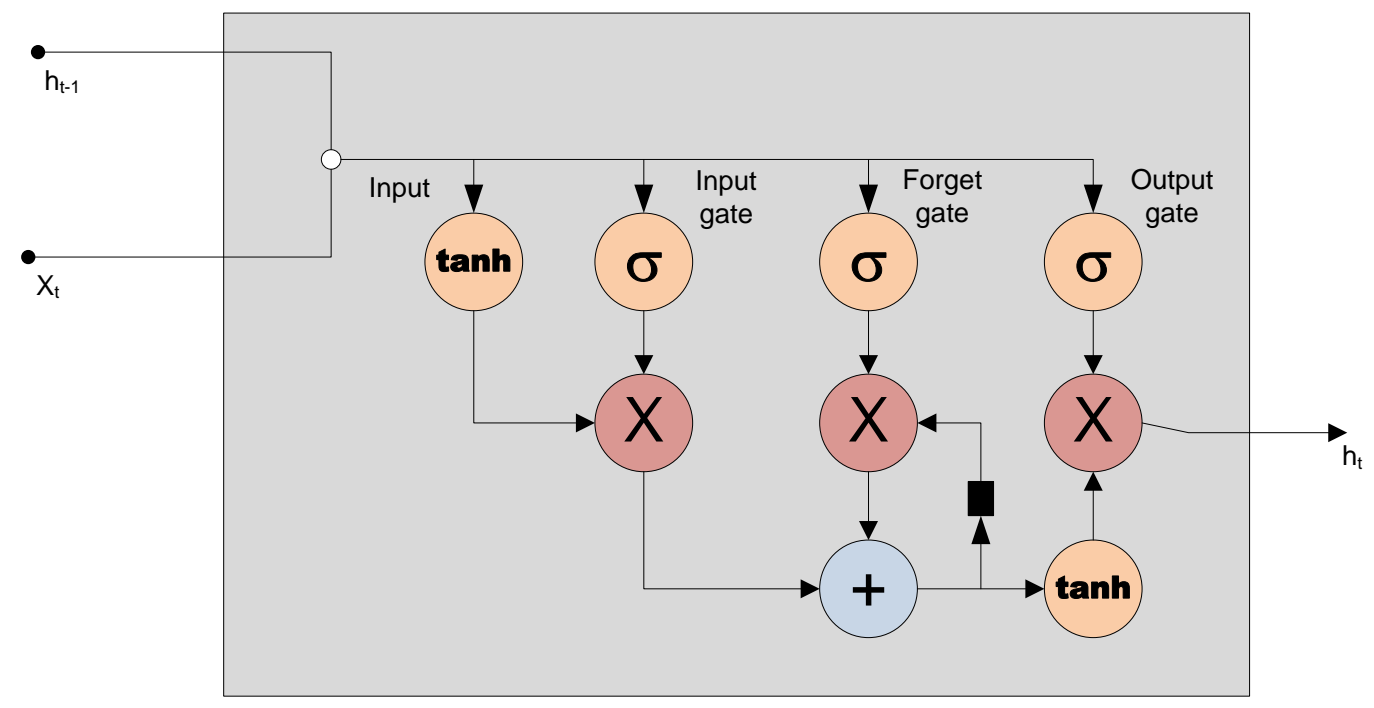

Figura 1. Célula de memória

Fonte: Adaptado de Thomas (2020).

A rede GRU (Gated Recurrent Unit) pode ser considerada uma variação da LSTM, porque ambas são projetadas de maneira semelhante. Na GRU são utilizadas apenas duas portas de controle. Uma porta de atualização (update gate) que faz o papel da porta de esquecimento e de entrada e uma porta de redefinição (reset gate). Por ser mais simples a 
rede GRU é mais rápida de treinar e precisa menos dados para generalizar (SILVA et al., 2020; DATA SCIENCE ACADEMY, 2020).

\section{Etapas do Trabalho:}

Inicialmente, neste trabalho, realizou-se a análise exploratória dos dados, obtidos do Operador Nacional do Sistema Elétrico (ONS, 2020), com a intenção de obter informações importantes sobre os mesmos. Em seguida, realizou-se a etapa de modelagem. Nesta etapa foram desenvolvidos os modelos de redes neurais LSTM e GRU.

Na sequência, fez-se a validação dos modelos das RNAs. Analisando-se a acurácia, dos modelos, com dados que não participaram do processo de treinamento e teste. Finalmente, na última etapa, realizou-se a previsão da geração eólica para 24 meses. Na Figura 2 apresentam-se as etapas realizadas para previsão da geração eólica da usina de Praia Formosa.

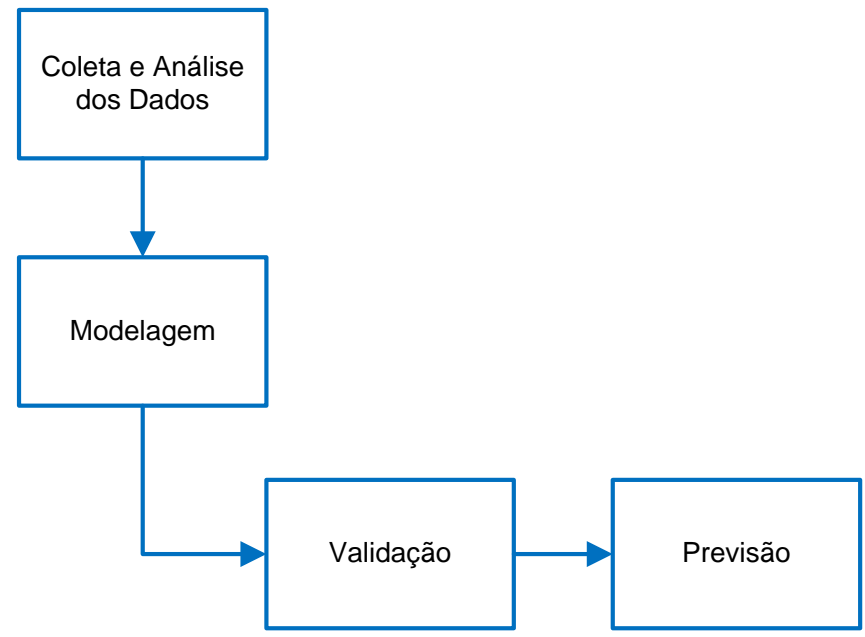

Figura 2. Etapas do trabalho

\section{Usina Eólica de Praia Formosa:}

A usina eólica de Praia Formosa (Figura 3) é um parque de produção de energia eólica localizado na cidade de Camocim no Ceará. Possui 50 aerogeradores, do modelo Suzlon S-88. Cada um com 2,1 MW de potência. Iniciou sua operação em 26 de agosto de 2009 (CAVALARI, 2016; COELHO, 2018). 


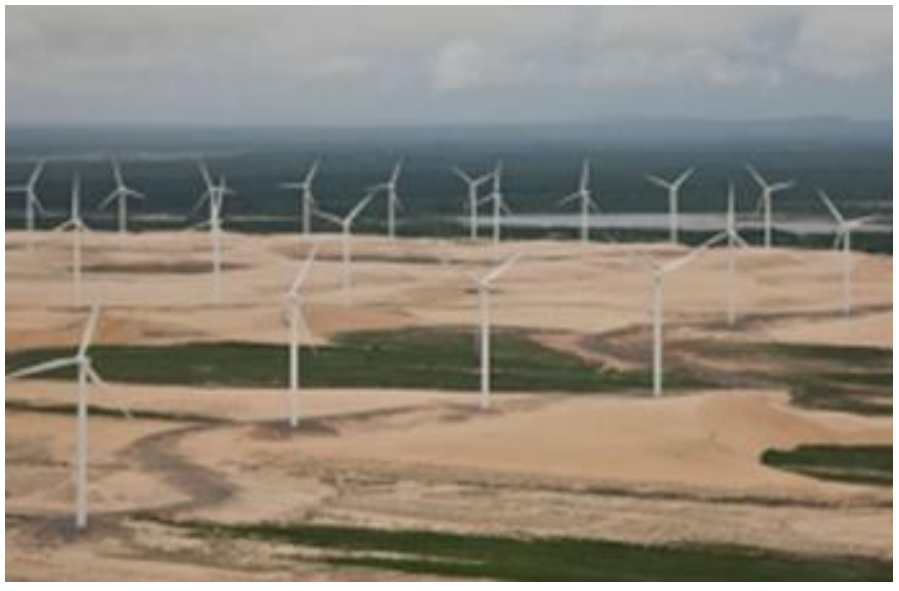

Figura 3. Usina de Praia Formosa Fonte: Cavalari (2016).

\section{Base de Dados:}

Para previsão da energia eólica, em MWmédio, da Usina de Praia Formosa, utilizouse uma base de dados com 117 meses (Jan/2011 - Set/2020) obtida da Operador Nacional do Sistema Elétrico (ONS, 2020). As potências relativas aos meses de Abril, Maio, Junho, Julho, Agosto e Setembro de 2020 foram retirados do conjunto de dados, para serem utilizados posteriormente para testes dos modelos. Os dados obtidos, da base de dados, já estavam limpos e sem a presença de outliers.

Os dez primeiros registros, do conjunto de dados da ONS, são apresentados na Tabela 1.

Tabela 1. Dez primeiros registros do conjunto de dados.

\begin{tabular}{cc}
\hline Data & Potência (MWmed) \\
\hline $2011-1$ & 12,4 \\
$2011-2$ & 12,7 \\
$2011-3$ & 6,7 \\
$2011-4$ & 4,7 \\
$2011-5$ & 5 \\
$2011-6$ & 11,6 \\
$2011-7$ & 15,8 \\
$2011-8$ & 35,7 \\
$2011-9$ & 37,5 \\
$2011-10$ & 42,2 \\
\hline \multicolumn{2}{c}{ Fonte: ONS (2020). }
\end{tabular}

A série histórica, obtida da ONS, é apresentada na Figura 4. 


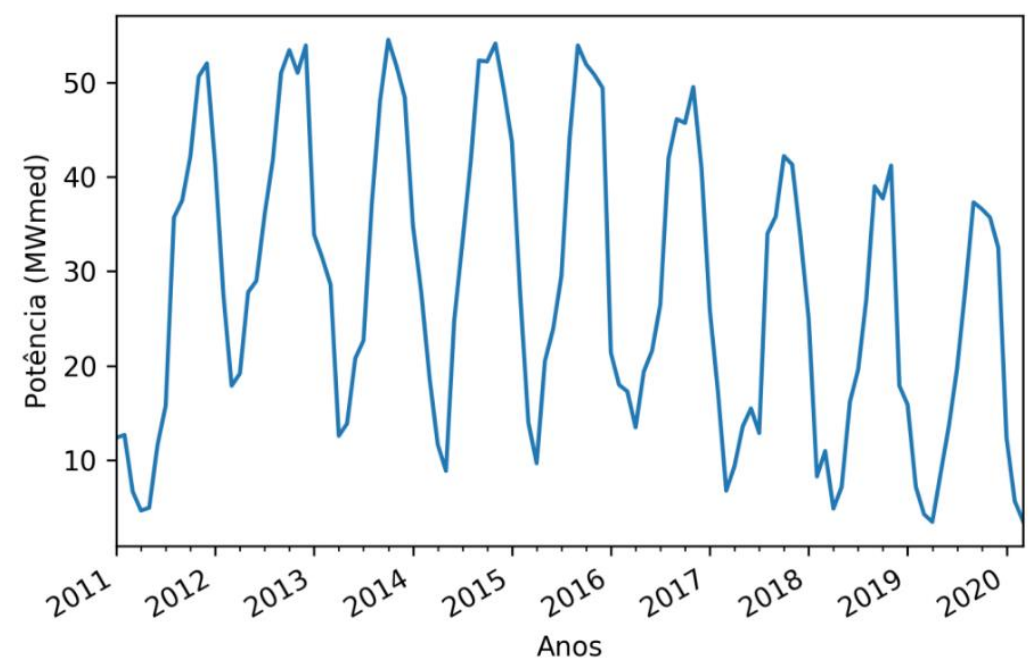

Figura 4. Série: geração eólica Fonte: ONS (2020).

Segundo Coelho (2018) o maior potencial eólico está concentrado durante os trimestres da primavera e do inverno. Este padrão foi também observado nos dados obtidos do Operador Nacional do Sistema Elétrico (Figura 5).

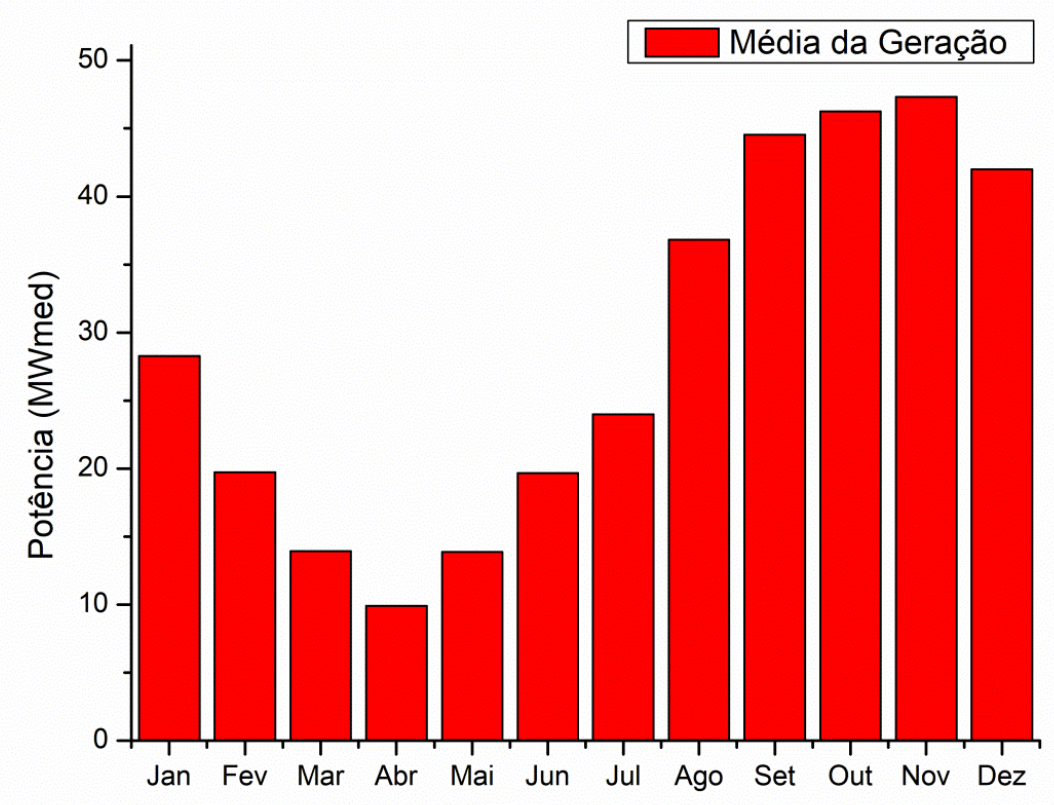

Figura 5. Média mensal da geração elétrica 


\section{Recursos:}

Modelos de previsão (LSTM e GRU) foram implementados na linguagem Python, utilizando o framework Keras. Keras é uma biblioteca para rede neural, de alto nível, escrita em Python e roda como frontend em TensorFlow ou Theano.

Os modelos foram executados por um notebook com o sistema Ubuntu 18.04. O notebook conta com um processador Intel(R) Core(TM) i7-26 e 16GB de memória RAM.

\section{Métricas:}

As métricas utilizadas, para avaliar a precisão, das previsões obtidas pelos algoritmos, são o Coeficiente de Determinação $\left(r^{2}\right)$, a Raiz Quadrada do Erro Médio Quadrático (RMSE) e o Erro Médio Absoluto (MAE). O r² indica quanto o modelo foi capaz de explicar os dados coletados. O RMSE apresenta o desvio padrão do erro de predição e o MAE representa o desvio médio entre os valores observados e preditos do modelo (CANKURT; SUBASI, 2015; PINHEIRO, 2020).

\section{RESULTADOS E DISCUSSÃO}

Inicialmente, neste trabalho, realizou-se uma análise descritiva dos dados (Tabela 2).

Tabela 2. Análise descritiva.

\begin{tabular}{cc}
\hline Parâmetro analisado & Potência (MWmed) \\
\hline Média (GWh) & 27,77 \\
\hline Mínimo (GWh) & 3,5 \\
\hline Máximo (GWh) & 54,5 \\
\hline Desvio Padrão (GWh) & 15,58 \\
\hline Coeficiente de Variação (\%) & 56,1 \\
\hline
\end{tabular}

Pode-se observar, dos dados apresentados na Tabela 2, que a geração, para o período em estudo, ficou em média de 27,77 MWmed. Apresentando, neste período, geração mínima e máxima de 3,5 MWmed e 54,5 MWmed, respectivamente.

Observa-se também, da Tabela 2, que o coeficiente de variação é $56.1 \%$, considerado muito alto, o que indica variabilidade dos dados. Segundo Pimentel (2009), se o coeficiente 
de variação for inferior a 10\% tem-se um coeficiente de variação baixo, de 10 a 20\% médio, de 20 a $30 \%$ alto e acima de $30 \%$ muito alto.

\section{Treinamento e Teste:}

Neste trabalho utilizou-se o método de fragmentação de Houldout onde a base de dados foi dividida com $67 \%$ dos dados para treinamento dos algoritmos e $33 \%$ para teste. Como critério de parada utilizou-se o método conhecido como Método de Parada Antecipada (Earling Stopping). Segundo Silva (2010), este método ajuda a projetar uma rede neural com bom poder de generalização.

Vários modelos de redes neurais, LSTM e GRU, foram testados utilizando como indicadores de desempenho: o coeficiente de determinação, o erro médio quadrático e erro médio absoluto. Os melhores modelos (Tabela 3) utilizam o algoritmo de otimização Adam com os seguintes hiperparâmetros: GRU neurons $=32$, LSTM neurons $=64$, batch $=6$, learning rate $=0.001$ e activate $=$ relu. Observa-se, que foi utilizado um look-back=24.

Tabela 3. Indicadores de desempenho (Teste).

\begin{tabular}{ccc}
\hline Métrica & LSTM & GRU \\
\hline $\mathbf{r}^{2}$ & 0.86 & 0.91 \\
RMSE & 4.9 & 4.0 \\
MAE & 3.46 & 2.53 \\
\hline
\end{tabular}

Pode-se observar, dos dados apresentados na Tabela 3, que o modelo GRU apresentou, para o conjunto de teste, maior $r^{2}$ e menores erros (RMSE e MAE).

Previsões:

Na sequência, realizaram-se previsões, da geração eólica, para os meses, de abril, maio, junho, julho, agosto e setembro de 2020, que não participaram da etapa de treinamento e teste (Tabela 4).

Tabela 4. Resultados das previsões (MWmed).

\begin{tabular}{cccc}
\hline Mês & ONS & GRU & LSTM \\
\hline $2020-04$ & 3.6 & 5.4 & 6.3 \\
$2020-05$ & 7.1 & 7.9 & 9.1 \\
$2020-06$ & 11 & 12.6 & 13.8 \\
$2020-07$ & 17.9 & 19.8 & 20.5
\end{tabular}




\begin{tabular}{llll}
$2020-08$ & 30.2 & 29.6 & 28.2 \\
$2020-09$ & 40.7 & 38.9 & 33.4 \\
\hline
\end{tabular}

Os resultados das previsões, em termos gráficos, são apresentados na Figura 6.

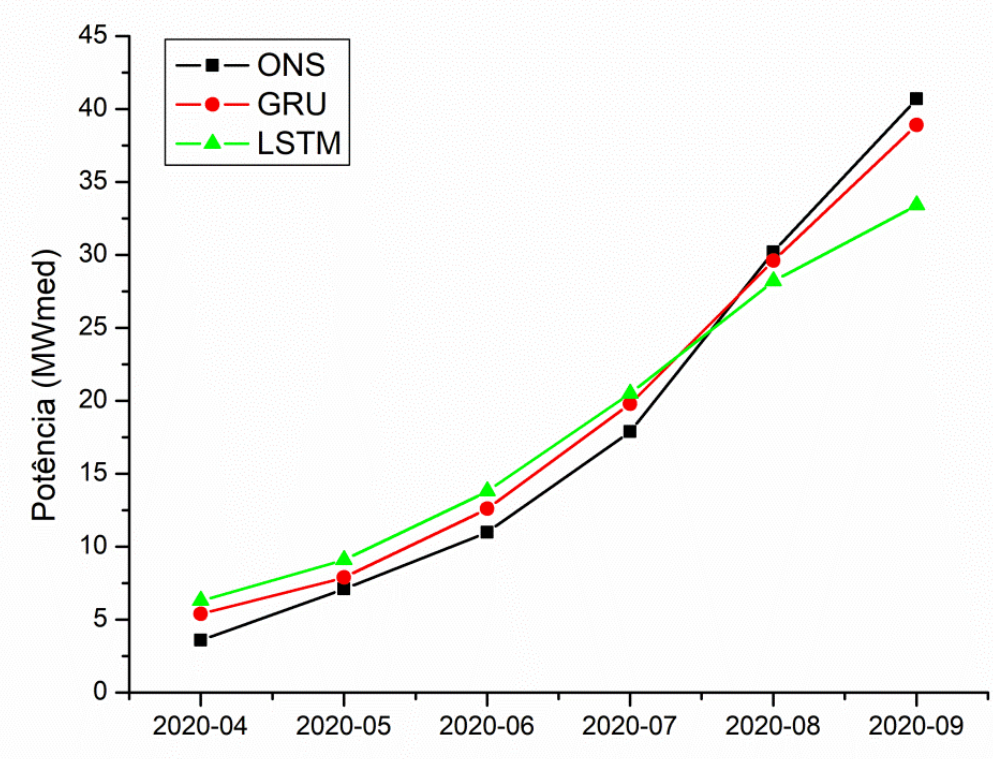

Figura 6. Resultados das previsões - abril, maio, junho, julho, agosto e setembro de 2020

Por meio dos resultados apresentados, na Tabela 4 e na Figura 5, conclui-se que o modelo GRU apresentou melhor desempenho que o modelo LSTM. Na Figura 7 apresentase, em vermelho, a previsão para 24 meses.

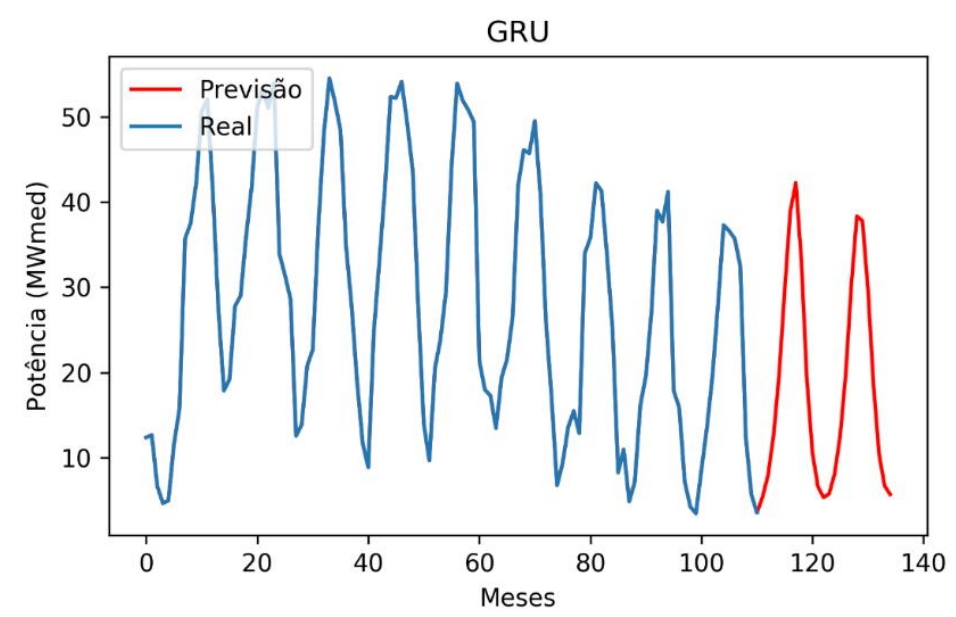

Figura 7. Previsão para 24 meses (04/2020 a 04/2022) 


\section{CONSIDERAÇÕES FINAIS}

Neste trabalho apresentou-se uma avaliação, de modelos de redes neurais artificiais, para previsão da geração de energia da usina eólica de Praia Formosa no Ceará. A série histórica de geração eólica, no período entre 2011 e 2020, foi fornecida pelo Operador Nacional do Sistema Elétrico (ONS). Os modelos, implementados neste trabalho, passaram pelas fases de: preparação de dados, definição das estruturas, estimativas, avaliação dos resultados e validação.

Inicialmente, realizou-se uma comparação entre o modelo LSTM e o modelo GRU, ambos implementados na linguagem Python. Observou-se que os indicadores $\left(r^{2}\right.$, RMSE e MAE) indicam, para o conjunto de teste, o melhor desempenho da rede GRU.

$\mathrm{Na}$ sequência, observou-se também, para os meses que não participaram do treinamento da rede (abril, maio, junho, julho, agosto e setembro de 2020), um melhor desempenho da rede GRU. Portanto, a proximidade entre valores preditos e reais demonstram a boa capacidade de generalização, para um horizonte de seis meses, do modelo GRU implementado neste trabalho.

\section{REFERÉNCIAS}

ARAUJO, W. C.; GOMES, E. S. Inovação tecnológica no uso das energias renováveis: o caso dos parques eólicos brasileiros. In: Anais do II Simpósio Nacional Espaço, Economia e Políticas Públicas. Anápolis, Góias, 2012.

BASTIANI, M.; SANTOS, J. A. A.; SCHMIDT, C. A P.; SEPULVEDA, G. P. L. Application of data mining algorithms in the management of the broiler production. Geintec, v. 8, 2018.

CANKURT, S.; SUBASI, A. Comparasion of linear regression and neural network models forecasting tourist arrivals to turkey. Eurasian Journal of Science \& Engineering. 2015.

CAVALARI, G. M. Avaliação de perdas elétricas devido ao ponto de interconexão do sistema de geração eólica na rede elétrica. 2016. Disponível em' https://www.ufjf.br/eletrica_energia/files/2016/09/TCC_GABRIEL-CAVALARI.pdf. Acesso em 15 nov. 2020.

COELHO, I. Estudo sobre a aplicação de redes neurais recorrentes para previsão da geração eólica e do preço da liquidação das diferenças. 2018. Disponível em: https://repositorio.ufsc.br/handle/123456789/192503. Acesso em 15 jul. 2020.

GRAVES A. Towards end-to-end speech recognition with recurrent neural networks. In: 31st International Conference on Machine Learning, Proceedings [...]. Beijing: ICML-14, 2014. 
LINS, F. G.; TORRES, A. J. Crescimento da geração eólica no Brasil: aprendizados para a operação do SIN. Disponível em: https://cenarioseolica.editorabrasilenergia.com.br/2020/02/28/crescimento-da-geracaoeolica-no-brasil-aprendizados-para-a-operacao-do-sistema-interligado-nacional/. Acesso em 15 nov. 2020.

NELSON, M. Q.; PEREIRA, A C. M.; OLIVEIRA R. A. Stock market's price prediction with LSTM neural networks. In: International Joint Conference of Neural Networks. Proceedings [...]. Anchorage: IJCNN , Alaska, 2017.

ONS, Operador Nacional do Sistema Elétrico. O sistema interligado nacional. Disponível em: http://www.ons.org.br. Acesso em 15 nov. 2020.

PIMENTEL, F. Curso de estatística experimental. Piracicaba: ESALQ, 2009.

PINHEIRO, T. C., SANTOS, J. A. A., PASA, L. A. Gestão da produção de frangos de corte por meio de redes neurais artificiais, Revista Holos, 2020.

SANTOS, J. A. A.; CHAUKOSKI, Y. Previsão do consume de energia elétrica na região sudeste: um estudo de caso usando SARIMA e LSTM. CEREUS, vol. 12, n. 4, 2020.

SILVA, M. P. Aplicação de redes neurais artificiais no diagnóstico de falha de turbina a gás. Dissertação (Mestrado em Engenharia Mecânica). Pontifícia Universidade Católica do Rio de Janeiro, Rio de Janeiro. 2010.

SILVA, M., et al. Previsão de carga horária como parâmetro do modelo de precificação e despacho hidrotérmico de curto prazo (DESSEM). Disponível em: http://abricom.org.br/eventos/cbic2019/cbic2019-96/. Acesso em: 12 jun. 2020.

THOMAS, A. Adventures in machine learning. Disponível em: https://adventuresinmachinelearning.com/category/deep-learning/lstms/. Acesso em: 12 mar. 2020.

YADAV, A.; JHA, C. K.; SHARAM, A. Optimizing LSTM for time series prediction in India stock market. Science Direct, 2020.

ZAO, Z. LSTM network: a deep learning approach for short-term traffic forecast. IET Intelligent Transport Systems, v. 11, 2017. CEREUS, vol. 12, n. 4, 2020. 